\title{
Spotted fever rickettsiosis in South Africa: Evaluation of laboratory diagnostic capacity and inter-laboratory comparison of serological testing
}

\author{
A N Trataris-Rebisz, ${ }^{1,2}$ MSc (Med); J Rossouw, ${ }^{1} \mathrm{PhD}$; W Markotter, ${ }^{3} \mathrm{PhD}$; J A Frean, ${ }^{1,4} \mathrm{MB}$ BCh, MMed (Path); J Weyer, ${ }^{1,3} \mathrm{PhD}, \mathrm{MPH}$ \\ ${ }^{1}$ Centre for Emerging Zoonotic and Parasitic Diseases, National Institute for Communicable Diseases, National Health Laboratory Service, \\ Johannesburg, South Africa \\ ${ }^{2}$ Department of Biochemistry, Genetics and Microbiology, Faculty of Agricultural Sciences, University of Pretoria, South Africa \\ ${ }^{3}$ Centre for Viral Zoonoses, Department of Medical Virology, School of Medicine, Faculty of Health Sciences, University of Pretoria, South Africa \\ ${ }^{4}$ Wits Research Institute for Malaria, School of Pathology, Faculty of Health Sciences, University of the Witwatersrand, Johannesburg, South Africa
}

Corresponding author: J Weyer (jacquelinew@nicd.ac.za)

\begin{abstract}
Background. Spotted fever rickettsiosis, also known as tick bite fever (TBF), is a common infectious disease in South Africa (SA). Although the diagnosis of TBF is often based on clinical grounds only, laboratory testing is important to confirm the diagnosis and can contribute to case management in the light of a myriad of differential diagnoses, and in complicated cases.

Objectives. To report on the availability and scope of laboratory tests for investigating suspected cases of TBF in SA, and the outcome of an inter-laboratory comparison (ILC) conducted for serological tests.

Methods. A self-administered questionnaire was circulated to major pathology laboratories in SA to determine what TBF tests they offered for TBF investigation. In addition, a clinical panel was provided to willing laboratories in order to perform an ILC of the serological tests. Results. Serological tests for TBF were available from five laboratories serving both the private and state medical sectors in SA. There was no standardised testing platform or result interpretation across the different laboratories. Polymerase chain reaction (PCR) tests were less frequently available, and not available to state-operated facilities. The outcome of the ILC indicated varied performance and interpretation of serological results for TBF.

Conclusions. Laboratory investigation for TBF is routinely and widely available in SA. Both serological and PCR-based methods were varied, and the lack of standardisation and interpretation of tests needs to be addressed to improve the overall quality of TBF diagnosis in SA. The utility of ILC to identify problem areas in serological testing for TBF is highlighted, and laboratories in SA are encouraged to use it to improve the quality of testing.
\end{abstract}

S Afr Med J 2019;109(4):223-226. DOI:10.7196/SAMJ.2019.v109i4.13788

The rickettsiae belong to a group of Gram-negative, arthropod-borne, obligate intracellular bacteria that cause a wide spectrum of disease, varying from mild and self-resolving to fatal infections. ${ }^{[1-4]}$ With the enhancement of diagnostic methodologies, specifically the use of molecular techniques, and improved ecological surveillance, the importance of rickettsial infections as neglected emerging infectious diseases globally has come to the fore. ${ }^{[2-7]}$ The rickettsiae are distributed globally and disease occurrence is associated with the distribution of arthropod vectors. ${ }^{[4]}$ The latter include various species of ticks, lice, fleas, mites and mosquitoes. ${ }^{[4]}$ There are currently three groups of diseases commonly identified as rickettsioses: (i) spotted fever group (SFG) and typhus group (TG) rickettsial infections; (ii) scrub typhus, associated with Orientia tsutsugamushi (formerly Rickettsia tsutsugamushi); and (iii) human ehrlichioses and anaplasmosis caused by pathogens in the family Anaplasmataceae. ${ }^{[3,4,8-10]}$ Rickettsioses do not have any specific pathognomonic signs, but rather a range of generalised signs and symptoms that may include fever, rash, lymphadenopathy and headache. ${ }^{[1]}$ These symptoms together with a history of an arthropod bite (usually a tick bite) $1-2$ weeks before the development of illness, in the presence or absence of a notable eschar (or tache noire), are considered highly suggestive of the diagnosis of rickettsiosis.
Spotted fever rickettsiosis, usually referred to as tick bite fever (TBF), is common in southern Africa. ${ }^{[12-16]}$ Seroprevalence studies have indicated up to $80 \%$ positivity in specific communities, while the disease is often reported in travellers to southern Africa. ${ }^{[17-19]}$ There are two main Rickettsia species associated with TBF in South Africa (SA), namely $R$. africae and $R$. conorii, causing African TBF and Mediterranean spotted fever, respectively. ${ }^{[16,18,20,21]} R$. africae is reportedly transmitted by Amblyomma (or 'bont') tick species, $A$. variegatum and $A$. hebraeum, whereas $R$. conorii has been associated with the brown dog tick, Rhipicephalus sanguineus. ${ }^{[16-18,21]}$ Single cases of TBF in SA associated with $R$. aeschlimannii (transmitted by $R$. appendiculatus $)^{[21]}$ and $R$. mongolotimonae have also been reported. ${ }^{[12]}$ The clinical presentation of TBF associated with these different rickettsiae is very similar, however, and identification of the aetiological agent is not required for patient management. ${ }^{[16,18,21]}$

The clinical syndrome of TBF is usually uneventful and resolves with antibiotics, but fatal cases have been reported in patients with and without comorbid features. ${ }^{[1,18]}$ Deaths are often associated with delayed clinical recognition and antibiotic treatment. Although clinical suspicion and patient response to doxycycline or tetracycline treatment are often used to diagnose cases of TBF, specific laboratory testing for $\mathrm{TBF}$ can be an important tool to direct patient management, especially 
in severe cases. ${ }^{[16,18,21]}$ The differential diagnosis of TBF is vast, including Crimean-Congo haemorrhagic fever, meningococcaemia, rubella, measles, secondary syphilis, disseminated gonococcal disease, enterovirus or arbovirus infections, anthrax, brucellosis, cat scratch disease, leptospirosis, typhoid, immune complex vasculitis or drug reactions, and the value of laboratory investigation should therefore not be underestimated. ${ }^{[16]}$

There are several factors to consider in the laboratory diagnosis of TBF. Conventional testing includes the Weil-Felix test, an agglutination assay detecting rickettsial antibodies in patient blood. ${ }^{[2]}$ This test is no longer recommended as it lacks greatly in sensitivity and specificity, but it is still practised in some routine laboratories. ${ }^{[16]}$ The indirect immunofluorescence assay (IFA) is considered the gold standard for TBF diagnosis and is the test most commonly performed in routine diagnostic laboratories. ${ }^{[22]}$ This form of testing is rapid and sensitive, but antibody cross-reactivity between Rickettsia species renders the test unsuitable for discerning the exact cause of the infection. Other proteobacterial antigens may also cross-react with rickettsial antigens, and this should be taken into consideration in the interpretation of results. Importantly, anti-rickettsial antibodies only develop after the first week of illness, and serological tests may therefore be uninformative during the acute and early stages of disease. Results need to be interpreted in the context of the patient's clinical history, since negative findings may inadvertently misdirect the diagnosis. ${ }^{[22]}$ IFA, based on microscopic observation, requires highly skilled laboratorians to interpret the reading of the assay. A highly sensitive and reproducible enzyme-linked immunosorbent assay (ELISA) for anti-rickettsia antibody detection is also commercially available, and allows for the differentiation of immunoglobulin G (IgG) and immunoglobulin $\mathrm{M}$ (IgM) antibodies. ${ }^{[22]}$ ELISAs have been shown to be more sensitive than IFAs in detecting low-level antibodies, and are suitable for demonstrating rising antibody titres. ${ }^{[22]}$ Molecular techniques are increasingly being used to detect rickettsial DNA in eschar swabs, skin biopsies or blood. ${ }^{[22,23]}$ These tests are, however, not as commonly available as serological testing. Culturing of rickettsiae is also not routinely available, as it requires the use of biosafety level 3 facilities and in vivo techniques. ${ }^{[6]}$ Other commercially available diagnostic tests include other agglutination assays, indirect haemagglutination, immunoperoxidase assay, complement fixation tests, Western immunoblotting and line probe assays. ${ }^{[22]}$ These tests vary in diagnostic sensitivity and specificity. In general, there is a lack of standardisation across the different testing platforms and between laboratories offering these tests. ${ }^{[22]}$

\section{Objectives}

To report on the status quo of laboratory testing for TBF in SA and on the findings of an inter-laboratory comparison (ILC) for serological tests.

\section{Methods \\ Study participants}

Five pathology service providers (hereafter laboratories) known to offer TBF testing were requested to participate in this study. They included laboratories servicing both the private and state healthcare sectors in SA.

\section{Questionnaire}

The study comprised two parts. The first part involved a questionnaire provided electronically to the laboratories, in order to determine the TBF testing available. Participation was voluntary and a period of 2 weeks was allowed for respondents to submit their answers. It was requested that senior, qualified staff complete the questionnaire. The self-administered questionnaire comprised 53 questions divided into five sections, requesting information on serological and molecular tests offered and technical details of the tests (available on request from the corresponding author). The quality assurance system applied was also interrogated. The data were subsequently captured and analysed in an Excel spreadsheet, version 2016 (Microsoft, USA).

\section{Inter-laboratory comparison}

The second part of the study was an ILC exercise to gauge the performance and agreement of serological results for TBF for a standardised clinical specimen panel. Laboratories indicated in the previously mentioned questionnaire whether they were willing to participate in an ILC exercise. Three of the five laboratories offering TBF testing agreed to take part. A single ILC panel was supplied to each laboratory and testing was performed through their routine protocols. Participating laboratories were requested to test the panel per routine protocol for TBF serological testing (for both anti-rickettsia IgG and IgM) and to report the results to the provider within 4 weeks of receipt.

The ILC testing panel comprised 10 human serum samples and was accompanied by a clinical and exposure scenario to guide interpretation of the results. The samples were derived from clinical material collected through previous research projects or clinical submissions to the Centre for Emerging Zoonotic and Parasitic Diseases, National Institute for Communicable Diseases. Ethical clearance for the use of the archival material was obtained prior to the study from the University of the Witwatersrand Human Research Ethics Committee (ref. no. M120667) (amended) and the University of Pretoria Human Research Ethics Committee (ref. no. 222/2017). The serum panel was characterised through repeat testing with four different commercially available assays for rickettsia IgG and IgM determination: Vircell IgG and IgM R. conorii IFA (Vircell, Spain), Vircell IgG and IgM R. conorii ELISA, Focus Diagnostics IgG and IgM SFG and TG IFA (Focus Diagnostics, USA), and Fuller Laboratories IgG and IgM SFG ELISA (Fuller Laboratories, USA). Testing was conducted four times (once per kit) without deviation from the manufacturers' guidelines, and the selection of samples included in the panel was based on consensus results of all four testing platforms. Four of the 10 samples included in the panel tested rickettsia IgG-positive, and the remaining six were IgG-negative. All 10 samples tested negative for IgM across all assays used. Only specimens that gave consistent, non-equivocal results were selected. Selected sera were homogenised by brief vortex mixing and centrifuged, followed by aliquoting and storage at $-20^{\circ} \mathrm{C}$. A subset of aliquots was subjected to stability testing, following storage at $4^{\circ} \mathrm{C}$ and $-20^{\circ} \mathrm{C}$, at 2-week intervals (i.e. distribution date, mid-point and closing of the ILC).

The panel was numerically coded with no identifiers linking a sample to patient information or the original study for which the samples were collected. The ILC panels were handled and transported on ice and in accordance with international regulations for the transport of diagnostic samples. Accompanying documentation included handling (including safety), storage and testing instructions, as well as a standardised response form.

Results reported were summarised in an Excel spreadsheet, version 2016. The agreement of results submitted by the participants with the expected result was calculated as a proportion of results consistent with the expected results. Likewise, the overall agreement of results for IgG and IgM, respectively, was calculated as the proportion of all results in agreement with the expected results.

\section{Resullts}

\section{Questionnaire}

All participating laboratories were compliant with the 2-week questionnaire and 4-week ILC result submission deadlines. 
Responses to the questionnaire were received from all five participating laboratories, representing both the private and state sectors of the SA healthcare system. Serological testing for antirickettsia IgG and IgM was offered by all the laboratories, with three of the laboratories indicating that they also offered polymerase chain reaction (PCR) tests. All laboratories offering rickettsia serology used commercially available IFA kits for IgG and IgM antibody detection. Four of the five laboratories used the Focus Diagnostics IFA kit and one used the Vircell IFA kit. All the serological tests were based on $R$. conorii antigens, although $R$. africae would also be detected owing to antigenic cross-reactivity. All respondents indicated that the commercial kits were subject to in-house validation for diagnostic use. Four of the five laboratories indicated that the rickettsial serological testing offered was accredited to international standards through the South African National Accreditation System. Three of the four accredited laboratories conducted routine proficiency testing through the Royal College of Pathologists of Australasia.

In addition to serology, three laboratories indicated that they also offered PCR to detect rickettsial DNA targets. Importantly, PCR testing was not available for state facilities. Of the three laboratories that offered PCR diagnostic assays, one used an in-house-developed method employing previously published primers, and the other two used a commercially available real-time PCR targeting the glt $A$ gene of all Rickettsia species. The laboratory that employed in-house methods had two real-time PCRs available, one that targeted the gltA gene for SFG rickettsiae and another that targeted the gltA gene for TG group rickettsiae. Various sample types (including blood, and eschar biopsies and swabs) may be tested using the commercial kit, but only plasma or serum was recommended for the in-house methods. Two of the three laboratories that offered rickettsial PCR used in-house-validated methods. None of the laboratories employed external proficiency testing or used accredited PCR methods.

\section{Inter-laboratory comparison}

Three laboratories participated in the serology ILC. Laboratory 1 used the Vircell IgG and IgM R. conorii IFA kit, while laboratories 2 and 3 used the Focus Diagnostics IgG and IgM IFA kit. The expected results (obtained using four different assays, described above) and the results obtained by the participants are summarised in Table 1. All participants reported using manufacturers' suggested protocols for the tests.

Overall agreement of results of $83 \%$ for IgG and $77 \%$ for IgM was achieved. Laboratories 2 and 3 reported false-positive IgG results for sample 2018/01-003, while all three participants reported false-positive results for sample 2018/01-010. No false-negative results were reported. For IgM, only laboratory 3 reported full agreement with expected results. Laboratories 1 and 2 collectively reported seven false-positive results for IgM. Half of the IgM results reported by laboratory 1 were false positive.

All three laboratories offered interpretation of negative and positive results in accordance with the kit manufacturers' recommendations. Importantly, this included wording that indicated that negative results do not exclude the diagnosis of TBF. Two of the laboratories indicated the importance of considering the testing of paired samples.

\section{Discussion}

TBF is a common disease in SA, and although diagnosis is often based on clinical presentation alone, laboratory confirmation is recommended to ensure that patient management is well directed. At the time of the present study, rickettsial serological testing was routinely available from five pathology providers in SA, of which three participated in the ILC exercise. Although the IFA is currently regarded as a gold-standard method for TBF diagnosis, the value of PCR testing on appropriate samples has been widely reported. Although not subject to ILC in this study, it was determined that PCR testing was available from three laboratories in SA. It was noteworthy that PCR-based testing was not available in state-operated facilities. In addition, only one of the laboratories recommended PCR as a supplement to serological testing in their interpretation of negative serological findings.

There was a good agreement of results for IgG testing across the panel, and participants interpreted these results in line with the recommendations of the commercial kit providers and knowledge regarding cross-reactivity among Rickettsia species, and the requirement

Table 1. Inter-laboratory comparison of results for rickettsia IgG and IgM tests

\begin{tabular}{|c|c|c|c|c|c|c|c|c|c|c|}
\hline \multirow{2}{*}{$\begin{array}{l}\text { Sample reference } \\
\text { number }\end{array}$} & \multirow{2}{*}{$\begin{array}{l}\text { Expected } \\
\text { IgG result }\end{array}$} & \multicolumn{3}{|c|}{ Participant IFA IgG results } & \multirow{2}{*}{$\begin{array}{l}\text { Agreement of } \\
\text { results for all } \\
\text { participants, \% }\end{array}$} & \multirow{2}{*}{$\begin{array}{l}\text { Expected } \\
\text { IgM result }\end{array}$} & \multicolumn{3}{|c|}{ Participant IFA IgM results } & \multirow{2}{*}{$\begin{array}{l}\text { Agreement of } \\
\text { results for all } \\
\text { participants, \% }\end{array}$} \\
\hline & & Lab $1^{*}$ & Lab $2^{\dagger}$ & Lab $3^{\dagger}$ & & & Lab $1^{*}$ & Lab $2^{\dagger}$ & Lab $3^{\dagger}$ & \\
\hline 2018/01-001 & $\mathrm{Neg}$ & $\mathrm{Neg}$ & $\mathrm{Neg}$ & $\mathrm{Neg}$ & 100 & Neg & Pos & Neg & $\mathrm{Neg}$ & 67 \\
\hline 2018/01-002 & Pos & Pos & Pos & Pos & 100 & Neg & Pos & Neg & $\mathrm{Neg}$ & 67 \\
\hline 2018/01-003 & Neg & $\mathrm{Neg}$ & Pos & Pos & 33 & Neg & $\mathrm{Neg}$ & Neg & Neg & 100 \\
\hline 2018/01-004 & Pos & Pos & Pos & Pos & 100 & Neg & Pos & $\mathrm{Neg}$ & $\mathrm{Neg}$ & 67 \\
\hline 2018/01-005 & Neg & $\mathrm{Neg}$ & $\mathrm{Neg}$ & Neg & 100 & Neg & $\mathrm{Neg}$ & Neg & $\mathrm{Neg}$ & 100 \\
\hline 2018/01-006 & $\mathrm{Neg}$ & Neg & $\mathrm{Neg}$ & $\mathrm{Neg}$ & 100 & Neg & Pos & Neg & Neg & 67 \\
\hline 2018/01-007 & Pos & Pos & Pos & Pos & 100 & Neg & $\mathrm{Neg}$ & Neg & $\mathrm{Neg}$ & 100 \\
\hline 2018/01-008 & Neg & $\mathrm{Neg}$ & Neg & Neg & 100 & $\mathrm{Neg}$ & $\mathrm{Neg}$ & Pos & $\mathrm{Neg}$ & 67 \\
\hline 2018/01-009 & Pos & Pos & Pos & Pos & 100 & $\mathrm{Neg}$ & Pos & Pos & $\mathrm{Neg}$ & 33 \\
\hline 2018/01-010 & $\mathrm{Neg}$ & Pos & Pos & Pos & 0 & $\mathrm{Neg}$ & $\mathrm{Neg}$ & Neg & $\mathrm{Neg}$ & 100 \\
\hline $\begin{array}{l}\text { Agreement } \\
\text { of results per } \\
\text { participant, \% }\end{array}$ & - & 90 & 80 & 80 & 83 & - & 50 & 80 & 100 & 77 \\
\hline
\end{tabular}


of follow-up testing for confirmation. The latter is important in the light of known high seroprevalence indicated in previous studies in SA, in order to elucidate previous v. current infections. A deficiency of the ILC exercise was that the measured serological titres or optical densities were not included in reporting and laboratories were expected to report only their final interpretation of the results. The titred results could have been useful to investigate the calling of false-positive results, particularly for sample 2018/01-010. The latter sample tested consistently negative by the panel provider with the two kits used by the participants, and also with an additional two commercial kits available at the time of the study.

The agreement of IgM results was less encouraging, with one of the participants reporting false-positive results for half of the panel. This laboratory used the Vircell IgG/IgM kit, while the other two participants used the kit supplied by Focus Diagnostics. Importantly, the samples tested repeatedly negative in the hands of the panel provider before and after the ILC exercise, using the Vircell kit but also the additional three commercial kits for IgM detection. To our knowledge, only one comparative study looking at commercially available rickettsia IFA kits has been published, which focused on comparison of the Focus Diagnostic IgG IFA kit with the Fuller Laboratories IgG IFA kit, and reported $100 \%$ agreement between the two kits when results were dichotomised in accordance with manufacturers' cut-offs. ${ }^{[24]}$ Our ILC participants indicated no deviation in the test protocol as recommended by the manufacturer, and included the initial IgG preabsorbing step, which is a common reason for false positives with IgM serological assays. As IFA is a microscopy-based method, the required subjective interpretation of visual observation may offer an explanation for the false-positive results (for example, with the observation of background autofluorescence). This subjectivity is generally regarded as a disadvantage of IFA methodology. In response to the ILC results, the laboratory in question followed up on the reporting of false-positive IgM results and concluded that they had indeed been overcalling background fluorescence, and have adjusted their protocol and training of staff accordingly.

Interestingly, none of the participants considered the use of the commercial IgM ELISA assay, which offers the advantage of objective result interpretation. In addition, laboratory 2 reported two falsepositive results. The rate of false-positive IgM findings was worrying, as these would support the incorrect diagnosis of TBF and could detract from the appropriate management of cases. A limitation of this study was the non-availability of IgM-positive serum samples, and this deficiency should be addressed in future ILC exercises to offer a more comprehensive analysis of interlaboratory performance.

The availability of proficiency testing panels for rickettsia serology globally is very limited, and owing to species diversity, which varies geographically, available panels may not represent the species of concern in a given region or country. The continued measure of performance across laboratories using well-characterised, local population-derived clinical material is therefore encouraged.

\section{Conclusions}

Currently there is no standardisation of laboratory diagnosis of TBF in $\mathrm{SA}$. The consequence is variable reliability of test results and possibly a devaluation of the role of laboratory confirmation of the disease. As commercial kits are used for serological diagnosis, the importance of local population-based validation to gain better understanding of test sensitivity and specificity in the SA context cannot be understated. Expanding the use of PCR could be an important contribution to investigation of suspected TBF cases, and again the use of validated methods subject to proficiency testing is encouraged.

To our knowledge, this is the first national ILC for rickettsia serology that has been conducted in SA. The value of continued ILC efforts for rickettsia serology, and also PCR, in SA is recognised and may contribute to the standardisation and improvement of the quality of laboratory investigations for TBF in the country.

Declaration. Publication was a requirement for AT-R's PhD degree. Acknowledgements. The authors acknowledge the contribution of the laboratories that participated in this study.

Author contributions. AT-R was the principal investigator of the study. All authors contributed to the study and the drafting of the manuscript.

Funding. The study was supported through institutional funding of the National Institute for Communicable Diseases and a National Health Laboratory Service (NHLS) Research Trust Development Grant (2015-1DEV43-RO01). AT-R was supported through postgraduate bursaries from the University of Pretoria, the National Research Foundation and the NHLS.

\section{Conflicts of interest. None.}

1. Lepidi H, Fournier PE, Raoult D. Histologic features and immunodetection of African tick-bite fever eschar. Emerg Infect Dis 2006;12(9):1332-1337. https://doi.org/10.3201/eid1209.051540

2. Blanton LS, Walker DH. Flea-borne rickettsioses and rickettsiae. Am J Trop Med Hyg 2017;96(1):53-56. https://doi.org/10.4269/ajtmh.16-0537

3. Parola P, Davoust B, Raoult D. Tick-and flea-borne rickettsial emerging zoonoses. Vet Res 2005;36(3):469492. https://doi.org/10.1051/vetres:2005004

4. Merhej V, Angelakis E, Socolovschi C, Raoult D. Genotyping, evolution and epidemiological findings of Rickettsia species. Infect Genet Evol 2014;25(Jul):122-137. https://doi.org/10.1016/j.meegid.2014.03.014 5. Essbauer S, Hofmann M, Kleinemeier C, Wölfel S, Matthee S. Rickettsia diversity in southern Africa: A small mammal perspective. Ticks Tick Borne Dis 2018;9(2):288-301. https://doi.org/10.1016/j. ttbdis.2017.11.002

6. Bizzini A, Péter O, Baud D, Edouard S, Meylan P, Greub G. Evaluation of a new serological test for the detection of anti-Coxiella and anti-Rickettsia antibodies. Microb Infect 2015;17(11):811-816. https://doi. $\mathrm{org} / 10.1016 /$ j.micinf.2015.09.015

7. Comer JA, PaddockCD, Childs JE Urban zoonoses caused by Bartonella, Coxiella, Ehrlichia, and Rickettsia species. Vector Borne Zoonotic Dis 2001;1(2):91-118. https://doi.org/10.1089/153036601316977714

8. Parola P, Socolovschi C, Jeanjean L, et al. Warmer weather linked to tick attack and emergence of severe 8. Parola P, Socolovschi C, Jeanjean L, et al. Warmer weather linked to tick attack and emergence
rickettsioses. PLoS Negl Trop Dis 2008:2(11):338. https://doi.org/10.1371/journal.pntd.0000338

9. Walker DH, Valbuena GA, Olano JP. Pathogenic mechanisms of diseases caused by Rickettsia. Ann N Y . Walker DH, Valbuena GA, Olano JP. Pathogenic mechanisms of diseases caused
Acad Sci 2003;990(1):1-11. https://doi.org/10.1111/j.1749-6632.2003.tb07331.x

Acad Sci 2003;990(1):1-11. https://doi.org/10.1111/j.1749-6632.2003.tb07331.x
0. Mahajan SK. Rickettsial diseases. J Assoc Physicians India 2012;60(Jul):37-44.

11. Faccini-Martínez ÁA, García-Álvarez L, Hidalgo M, Oteo JA. Syndromic classification of rickettsioses: An approach for clinical practice. Int J Infect Dis 2014;28(Nov):126-139. https://doi.org/10.1016/j. ijid.2014.05.025

12. Pretorius AM, Birtles RJ. Rickettsia mongolotimonae infection in South Africa. Emerg Infect Dis 2004;10(1):125-126. https://doi.org/10.3201/eid0603.000309

13. Ndip LM, Bouyer DH, Travassos Da Rosa AP, et al. Acute spotted fever rickettsiosis among febrile patients, Cameroon. Emerg Infect Dis 2004;10(3):432-437. https://doi.org/10.3201/eid1003.020713

14. Raoult D, Fournier PE, Fenollar F, et al. Rickettsia africae, a tick-borne pathogen in travelers to subSaharan Africa. N Engl J Med 2001;344(20):1504-1510. https://doi.org/10.1056/nejm200105173442003

15. Ndip LM, Biswas HH, Nfonsam LE, et al. Risk factors for African tick-bite fever in rural central Africa. Am J Trop Med Hyg 2011;84(4):608-613. https://doi.org/10.4269/attmh.2011.10-0191

16. Frean J, Blumberg L. Ogunbanjo GA. Tick bite fever in South Africa. S Afr Fam Pract 2008;50(2):33-35. . Frean J, Blumberg L. Ogunbanjo GA. Tick bite fever

17. Socolovschi C, Renvoisé A, Brouqui P, Parola P, Raoult D. The use of eschar swabs for the diagnosis ofolovschi C, Renvoise A, Brouqui P, Parola P, Raoult D. The use of eschar swabs for the diagnosis
of African tick-bite fever. Ticks Tick Borne Dis 2012;3(5):361-363. https://doi.org/10.1016/j. ttbdis.2012.10.018

18. Jensenius M, Fournier P, Kelly P, Myrvang B, Raoult D. African tick bite fever. Lancet Infect Dis 2003;3(9):557-564. https://doi.org/10.1016//1473-3099(03)00739-4

19. Simpson GJ, Quan V, Frean J, et al. Prevalence of selected zoonotic diseases and risk factors at a human-wildlife-livestock interface in Mpumalanga Province, South Africa. Vector Borne Zoonotic Dis 2018;18(6):303-310. https://doi.org/10.1089/vbz.2017.2158

20. Jensenius $\mathrm{M}$, Fournier PE, Vene $\mathrm{S}$, et al. African tick bite fever in travelers to rural sub-Equatorial Africa. Clin Infect Dis 2003;36(11):1411-1417. https://doi.org/10.1086/375083

21. Pretorius AM, Birtles RJ. Rickettsia aeschlimannii: A new pathogenic spotted fever group rickettsia, South Africa. Emerg Infect Dis 2002;8(8):874. https://doi.org/10.3201/eid0808.020199

22. La Scola B, Raoult D. Laboratory diagnosis of rickettsioses: Current approaches to diagnosis of old and na Scola B, Raoult D. Laboratory diagnosis of rickettsioses: Curre
new rickettsial diseases. J Clin Microbiol 1997;35(11):2715-2727.

23. Portillo A, de Sousa R, Santibáñez S, et al. Guidelines for the detection of Rickettsia spp. Vector Borne Portillo A, de Sousa R, Santibáñez S, et al. Guidelines for the detection
Zoonotic Dis 2017;17(1):23-32. https://doi.org/10.1089/vbz.2016.1966

24. Kantsø B, Svendsen CB, Jørgensen CS, Krogfelt KA. Evaluation of serological tests for the diagnosis of rickettsiosis in Denmark. J Microbiol Methods 2009;76(3):285-288. https://doi.org/10.1016/j. mimet.2008.12.012 